\title{
ORIGINAL
}

\section{IMPACTO DEL TRATAMIENTO CON TERIPARATIDA EN LA CALIDAD DE VIDA DE LAS PERSONAS CON OSTEOPOROSIS}

\author{
Marta Osca Guadalajara (1), Natividad Guadalajara Olmeda (2) y Rosario Escartín Martínez (1).
}

(1) Unidad del dolor. Servicio de Anestesiología y Reanimación. Hospital Obispo Polanco. Teruel.

(2) Departamento de Economía y Ciencias Sociales. Universitat Politècnica de València. Valencia.

Para la elaboración del presente trabajo no se contó con ninguna beca ni ayuda económica.

No existen conflictos de interés.

\section{RESUMEN}

Fundamentos: La pérdida ósea en pacientes osteoporóticos, conlleva riesgo de fracturas, dolor óseo vertebral y disminución de la calidad de vida. El objetivo de este trabajo fue analizar el efecto de la teriparatida (TPTD) en pacientes osteoporóticos y con dolor vertebral.

Métodos: Estudio observacional longitudinal prospectivo, entre abril de 2006 y febrero de 2014, en los 77 pacientes tratados con TPTD en la Unidad del Dolor del Hospital de Teruel. La duración del tratamiento fue de $18 \mathrm{o}$ 24 meses. Se utilizó la Escala Visual Analógica (EVA) para la medición del dolor y el cuestionario europeo de calidad de vida (EuroQol-5D) para obtener la tarifa social (TS), antes y después el tratamiento. Se realizó un análisis descriptivo, de regresión lineal y logística.

Resultados: Se observó una mejoría del dolor (80\%) y de la calidad de vida (65\%). Se mejoró la EVA media (5,42 a 3,47 puntos) y el EuroQol-5D ( 0,36 a 0,58 puntos). La regresión indicó una mejora de la EVA en 0,441 puntos por cada punto de EVA inicial, y de la TS en 0,0528 puntos por cada 0,1 puntos de TS inicial. La probabilidad de mejorar la EVA en 3 puntos $(\mathrm{OR}=2,021)$, fue mayor que de mejorar 2 puntos $(\mathrm{OR}=1,695)$.

Conclusiones: La TPTD en pacientes osteoporóticos reduce el dolor óseo y mejora la calidad de vida. Su efecto es mayor en pacientes con peor estado de salud inicial, pudiendo ser utilizado como criterio para las decisiones terapéuticas y de gestión clínica.

Palabras clave: Calidad de vida. Dolor de espalda. Osteoporosis. Teriparatido.

\section{Correspondencia}

Marta Osca Guadalajara

C/Ruiz Jarabo s/n CP 44002 Teruel (ESPAÑA)

Teléfono 659909821

email: mosca@salud.aragon.es

\section{ABSTRACT Impact of Teriparatide on Quality of Life in Osteoporotic Patients}

Background: Lost bone in osteoporotic patients increases the risk of fractures and back pain, and decreases quality of life. The aim of this study was to analyse the effect of teriparatide (TPTD) in osteoporotic patients with vertebral pain.

Methods: A prospective observational study between April 2006 and February 2014 was done with 77 patients treated with teriparatide in the Pain Unit of Hospital Obispo Polanco of Teruel (Spain). Treatment duration was 18 or 24 months. Pain was assessed by the Visual Analogue Scale (VAS). Health-related quality of life was measured using the European Quality of Life Questionnaire (EuroQol-5D) in order to obtain the social tariff (ST). Pre and post- treatment values were collected respectively. A descriptive and regression analysis was done.

Results: Improvement in pain was observed $(80 \%)$ and in health-related quality of life (65\%). The mean VAS improved (from 5.42 to 3.47 points) and the mean health status value too (from 0.36 to 0.58 points). The regression indicated an improvement of VAS in 0.441 for each initial VAS point, and of ST in 0.0528 points for each 0.1 initial ST point. The probability of VAS reduction in 3 points $(\mathrm{OR}=2.021)$ was greater than in 2 points $(\mathrm{OR}=1.695)$

Conclusions: TPTD reduces pain and improves quality of life of osteoporotic patients. The worse the baseline situation, the more patients' health improved, so it could be used as criteria for therapeutic decisions and health management.

Keywords: Back pain. Osteoporosis. Quality of life. Teriparatide.

DOI: 


\section{INTRODUCCIÓN}

La osteoporosis es una enfermedad metabólica generalizada que se caracteriza por la pérdida de la cantidad y la calidad de hueso. Provoca dolor de espalda y su mayor consecuencia es el aumento del riesgo de sufrir una fractura ósea, produciendo un aumento del coste socio-sanitario, mayor morbilidad y mortalidad, junto a un incremento del dolor y disminución de la calidad de vida ${ }^{1-6}$.

Existen muchos factores de riesgo de la osteoporosis, modificables y no modificables. La edad es un factor de riesgo no modificable muy relacionado en el sexo femenino con la menopausia. En las personas mayores de 70 años tiende a equilibrarse la proporción mujeres/hombres, de manera que en las menores de 75 años padecen la enfermedad seis mujeres por cada hombre y a partir de los 75 años dos mujeres por cada hombre?

En España padecen osteoporosis aproximadamente tres millones de personas, de las cuales 2,5 millones son mujeres ${ }^{8}$. Sin embargo, sólo el 18\% de los pacientes están diagnosticados y se prevé un aumento de la incidencia, a causa del envejecimiento de la población. Por lo tanto, la osteoporosis constituye un problema importante no sólo para la salud de los pacientes sino también para la salud pública9 .

En otros países europeos, como Francia, se estima un aumento del $15 \%$ de mujeres osteoporóticas entre 2010 y $2020^{10}$. En Suecia se producen 70.000 fracturas de tipo osteoporótico al año de las cuales el $27 \%$ son de cadera y el $23 \%$ vertebrales. Se estima que en 2015 en este país se produzca un incremento de 1,1 millones de fracturas de cadera en hombres ${ }^{11}$. En EeuU la población osteoporótica era de 10 millones de personas en 2005 y se calcula que llegará a alcanzar los 14 millones en 2020, cuyo coste asociado a fracturas se incrementará de 17 billones de euros a más de 22 en dicho periodo ${ }^{1}$.
Uno de los objetivos de los gestores de salud es la prevención de la enfermedad, ya sea primaria (aparición de nuevos casos), secundaria (controlar la evolución de casos existentes) y terciaria (reducir las incapacidades derivadas de la enfermedad). Para ello, se adoptan medidas farmacológicas y no farmacológicas relacionadas con los hábitos de vida ${ }^{2}$.

Existen diferentes fármacos para el tratamiento de la osteoporosis, entre ellos la teriparatida [rh 1-34 PTH] (TPTD). Se trata de un análogo de la hormona paratiroidea humana (PTH) y está formado por su porción activa, concretamente por la secuencia de aminoácidos 1-34. Es un inductor óseo que tiene efectos positivos en la microarquitectura del hueso, promoviendo el aumento de su densidad y fuerza ${ }^{12,13}$. También ayuda a la osteointegración de los implantes de titanio y es una terapia efectiva en los defectos óseos periodontales de la osteonecrosis relacionada con los bifosfonatos ${ }^{14}$.

La TPTD está indicada en el tratamiento de la osteoporosis en mujeres posmenopáusicas ${ }^{1}$ y en varones con un aumento del riesgo de fractura. En mujeres posmenopáusicas se ha demostrado una disminución significativa en la incidencia de fracturas vertebrales y no vertebrales, pero no en fracturas de cadera.

También se utiliza en el tratamiento de la osteoporosis asociada a terapia sistémica mantenida con glucocorticoides, en mujeres $\mathrm{y}$ hombres con un incremento del riesgo de fractura ${ }^{15}$.

Está demostrada su utilidad en la disminución del dolor vertebral en pacientes osteoporóticos y en la mejoría de la calidad de vida. Además, es eficaz como osteoformador tras tratamiento quirúrgico en las fracturas periprotésicas y en fracturas con retardo en la consolidación ${ }^{16,17}$.

La dosis de fármaco recomendada son 20 $\mu \mathrm{g}$ diarios administrados por vía subcutá- 
nea. Inicialmente la duración aconsejada del tratamiento fue de 18 meses pero estudios posteriores consideraron más adecuada una duración de $24^{18,19}$.

Desde que se aprobó por primera vez en EEUU en 2002 y en la Unión Europea en 2003, para el tratamiento de la osteoporosis en hombres y mujeres, la TPTD es una alternativa al uso de agentes antiresortivos combinada con calcio y vitamina $\mathrm{D}^{3}$. Además, no se han encontrado diferencias en los efectos de la TPTD entre poblaciones japonesas y caucásicas ${ }^{13}$.

Diversos trabajos recientes realizados en diferentes países han analizado el efecto de la TPTD en la reducción del riesgo de fractu$\operatorname{ras}^{4,20,21}$ y del dolor, así como en la mejoría de la calidad de vida ${ }^{5,6,10,11,18,22-24}$.

El objetivo del presente estudio es realizar una valoración analgésica, de la calidad de vida y del riesgo de fracturas tras recibir tratamiento con TPTD subcutánea en pacientes con osteoporosis y dolor vertebral.

\section{MATERIAL Y MÉTODOS}

Estudio observacional longitudinal prospectivo con 77 pacientes tratados en la Unidad del dolor del Hospital Obispo Polanco de Teruel (España). Teruel presenta uno de los índices más altos de vejez de España ${ }^{25}$ (24\% de la población mayor de 65 años).

Los criterios de inclusión en el estudio fueron todos los pacientes que presentaron dolor vertebral con una Escala Visual Analógica (EVA) $\geq 3$, con factores de riesgo (historia familiar de fractura de cadera, edad, sexo, índice de masa corporal $\left(<19 \mathrm{~kg} / \mathrm{m}^{2}\right)$, menopausia precoz primaria o secundaria (ooforectomía bilateral u otras causas) y tratamiento de más de 5 años con bifosfonatos, debido al aumento del riesgo de osteonecrosis) y una de las siguientes características:

- Densitometría ósea significativa de osteoporosis (T-score $<-2,5$ desviaciones es- tándar en columna o cadera), que diagnostica la osteoporosis menos severa.

- Signos radiológicos de disminución de la densidad ósea (hipertransparencia ósea, disminución/deformidad altura vertebral).

- Fractura por fragilidad (aplastamiento vertebral, fractura distal del radio, fractura proximal de fémur). Muestran la osteoporosis más severa.

Las diferencias de diagnóstico utilizadas fueron debidas a criterio médico en función de las características clínicas del paciente y de la disponibilidad de pruebas. Todos fueron tratados con TPTD en el periodo comprendido entre abril 2006 y febrero de 2014, durante 18 meses en los primeros años del periodo (2006-2009) y durante 24 meses en los últimos años (2010-2014). La diferente duración fue debida a las indicaciones de las investigaciones sobre la duración del tratamiento, publicadas a lo largo del estudio ${ }^{18,19}$.

Se administró a todos ellos una dosis de $20 \mu \mathrm{g}$ diaria por vía subcutánea. Los pacientes tratados sin indicación correcta del fármaco, los que no habían finalizado el tratamiento y las pérdidas por fallecimiento durante el seguimiento fueron excluidos del estudio, por lo que finalmente se incluyó a 49 sujetos.

Su edad osciló entre los 57 y los 94 años y $47(96 \%)$ fueron mujeres. Como pruebas complementarias de imagen a 36 pacientes se les realizó radiografía de cadera y columna y a otros 13 densitometría ósea, presentando un T-score medio de $-2,12$ y $-2,86$ en cadera y columna respectivamente.

Se utilizó la EVA para la valoración del dolor, que se puntúa desde 0 (no dolor) a 10 (máximo dolor percibido). Se consideraron los valores de 0-3 como dolor leve, de 3-6 dolor moderado y de 7-10 dolor fuerte, registrándose los de cada uno de los pacientes en las fases previa y posterior a la toma del fármaco. 
Para la medición de la calidad de vida relacionada con la salud (CVRS) se utilizó el cuestionario europeo EQ-5D (anteriormente EuroQol), ampliamente utilizado en estudios sobre TPTD ${ }^{5,6,10,23,24}$ por su sencillez y rapidez para la obtención de datos, así como por su utilidad en la asignación de recursos sanitarios.

Con el EQ-5D ${ }^{26}$ el sujeto valora su estado de salud primero en niveles de gravedad por dimensiones (sistema descriptivo) a través de un cuestionario y luego en una escala visual analógica global. Un tercer elemento del EQ-5D es el índice de valores sociales: tarifa social (TS), que se obtiene para cada estado de salud creado por el instrumento. El sistema descriptivo contiene cinco dimensiones de salud (movilidad, cuidado personal, actividades cotidianas, dolor/malestar y ansiedad/depresión), cada una de las cuales se divide en tres niveles de gravedad (ausencia de problemas, algunos problemas o moderados y problemas graves). Posteriormente se codifican los niveles de gravedad y se utiliza el algoritmo de cálculo del Reino Unido, cuyos coeficientes fueron adaptados para el cálculo de la TS en España, lo que permite realizar comparaciones entre diferentes países. Los valores de TS oscilan entre 1 (mejor estado de salud) y 0 (la muerte), aunque existen valores negativos que se categorizan como peores que la muerte ${ }^{27}$.

Para el análisis estadístico se utilizaron las técnicas de regresión lineal ${ }^{13,28,29}$ y de regresión logística ${ }^{1,4,18,20,21,23}$ con el uso del SPSS para Windows versión 16.

En la técnica de regresión lineal por mínimo cuadrados ordinarios, las variables a explicar fueron dos:

- Mejoría del dolor según EVA (MEVA), definida como la diferencia entre la EVA previa y la EVA posterior, de manera que toma valores positivos cuando hay una mejora del dolor.
- Mejora de la TS (MTS), definida como la diferencia entre la TS posterior y la previa. Toma valores positivos cuando hay una mejoría de la calidad de vida.

La bondad del análisis fue estimada por el $\mathrm{R}^{2}$ corregido y el test de F de Snedecor, mientras que los valores de la t de Student, para cada coeficiente $\beta$ de la variable explicativa, determinaron su nivel de significación (p).

Con la técnica de regresión logística se obtuvieron las probabilidades de mejoría de la salud y el riesgo de sufrir una fractura tras la administración de la TPTD. En esta técnica se explicaron tres variables binarias definidas a partir de la variable MEVA: MEVA $>2$, MEVA $>3$ y MEVA $>4$, que se corresponden con una mejoría de la EVA en más de 2, 3 y 4 puntos, respectivamente. Y una cuarta variable binaria correspondiente a la incidencia de fractura después del tratamiento.

La bondad de los odds ratio (OR) se estimó a través de la chi $^{2}$ (prueba de Wald) y su valor $\mathrm{p}$ asociado, y la del ajuste con el logaritmo del coeficiente de verosimilitud.

Las variables explicativas utilizadas en ambas técnicas (lineal y logística) fueron:

- Seis de tipo cuantitativo: EVA previa, EVA posterior, TS previa, TS posterior, edad $\mathrm{y}$ duración del tratamiento.

- Cinco cualitativas binarias: existencia de fractura previa, existencia de fractura posterior y tipo de fractura: vertebral, fémur o radio.

\section{RESULTADOS}

Se observó una mejoría clínica del dolor óseo en 39 (80\%) pacientes tras el tratamiento. La EVA media \pm desviación típica del total de pacientes se redujo de 5,42 $\pm 1,696$ a $3,47 \pm 1,549$ puntos, después del tratamiento (figura 1).

Igualmente la TS media \pm desviación típica se incrementó de 0,358+0,271 a 


\section{Figura 1}

\section{Puntuaciones en la Escala Visual Analógica. Previa y posterior al tratamiento}
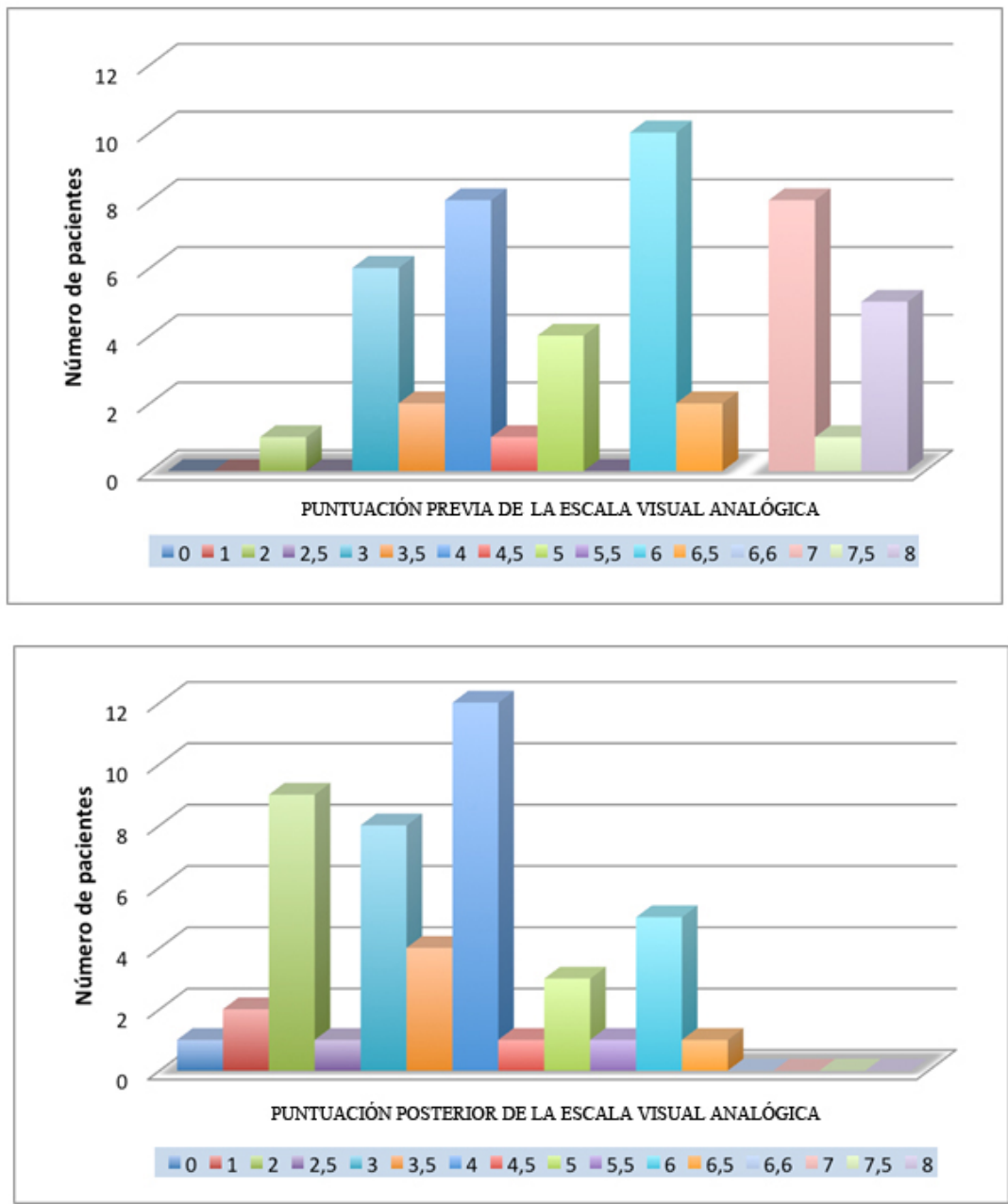

0,583+0,348 puntos (figura 2), produciéndose también una mejoría de la calidad de vida en 32 de los pacientes $(65 \%)$.

El número de pacientes que sufrió alguna fractura antes de la administración del fármaco fueron $30(61 \%)$, siendo $24(80 \%)$ fracturas vertebrales. De estos 30 pacientes, 21 estaban siendo tratados con calcio y bi- fosfonatos en el momento de la fractura, 4 con ranelato de estroncio, 1 con denosumab y el resto no recibía medicación alguna. Tras la finalización del tratamiento y hasta febrero de 2014, sólo 7 (23\%) pacientes sufrieron alguna fractura. De los 19 (39\%) restantes que no presentaron alguna fractura antes del tratamiento, 5 (26\%) tuvieron fractura (tabla 1). 

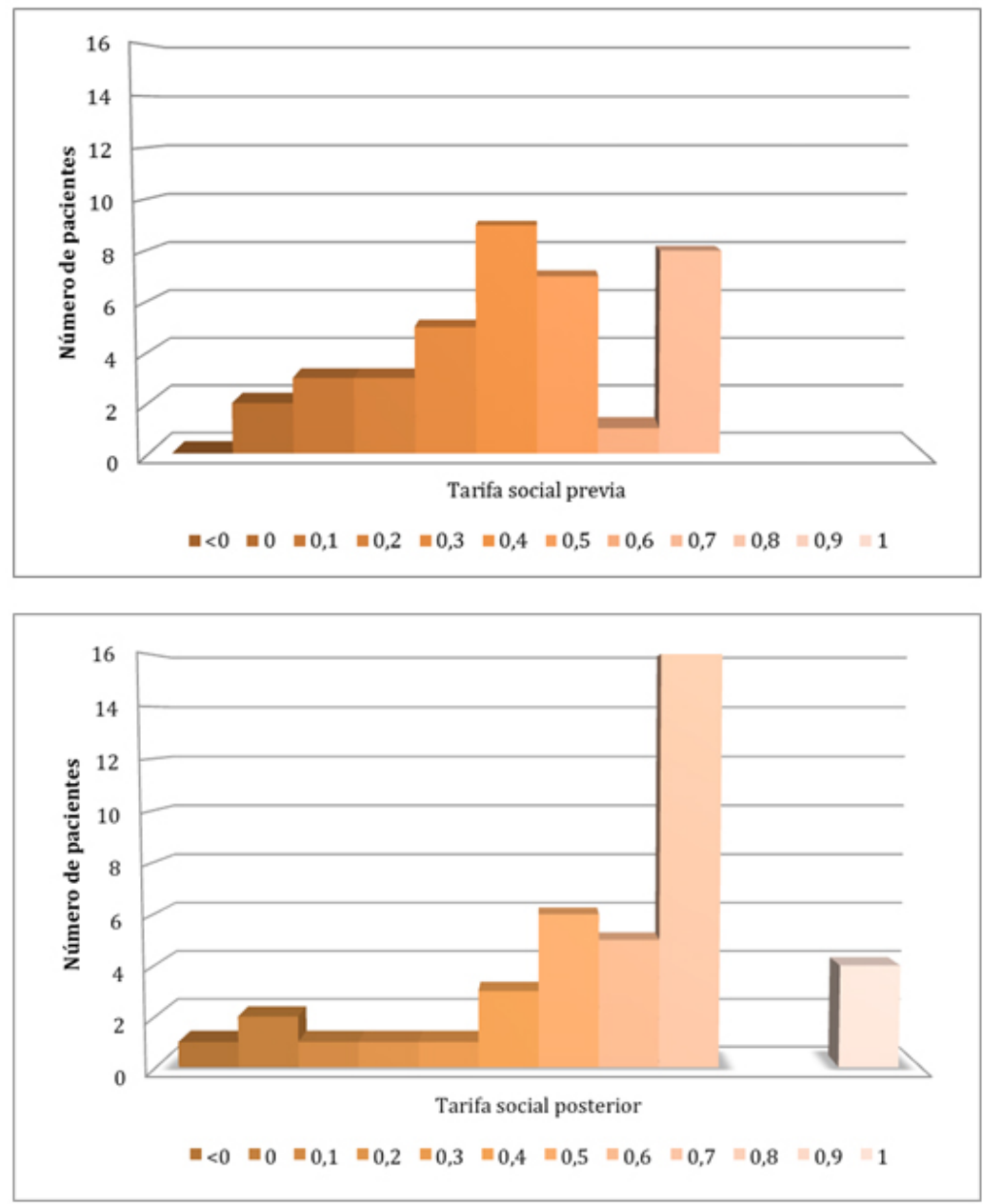

En los resultados de la regresión lineal (tabla 2) el coeficiente de la variable explicativa EVA previa (EVAPREV) de la MEVA es positivo, tanto para el total de pacientes $(0,441$ puntos $(\mathrm{p}$ valor $=0,000)$ ), como para los que habían sufrido fractura previa $(0,455)$ o no $(0,419)$. Esto significa que a mayor EVAPREV (mayor dolor) la mejoría del dolor es mayor; por cada punto de EVAPREV, el dolor mejora o se reduce en $0,441,0,455$ y 0,419 puntos, respectivamente.
El coeficiente de la TS previa (TSPREV) que explica la MTS es negativo en todos los casos, de $-0,528$ ( $p=0,004),-0,372$ y $-0,750$ en el mismo orden. Esto indica que en los pacientes con peor estado inicial (TSPREV más baja), la MTS es mayor que en pacientes con mejor estado de salud inicial (TSPREV más alta). La TS aumenta en 0,0528, 0,0372 y 0,0750 puntos por cada 0,1 puntos de TSPREV, respectivamente. 


\begin{tabular}{|c|c|c|c|c|}
\hline \multicolumn{5}{|c|}{$\begin{array}{c}\text { Tabla } 1 \\
\text { Distribución de pacientes según la mejoría } \\
\text { de la EVA y de la TS, } \\
\text { la incidencia de fracturas y la edad media }\end{array}$} \\
\hline & & \multicolumn{2}{|c|}{ Fractura previa } & \multirow[b]{2}{*}{ Total } \\
\hline $\begin{array}{l}\text { Mejoría } \\
\text { de la EVA }\end{array}$ & $\begin{array}{l}\text { Fractura } \\
\text { posterior }\end{array}$ & $\mathrm{Si}$ & No & \\
\hline \multirow{3}{*}{$\begin{array}{l}\text { No } \\
\mathrm{n}=10\end{array}$} & $\mathrm{Si}$ & 2 (75 años) & 2 (72,5 años) & 4 \\
\hline & No & 5 (72,3 años) & 1 (64 años) & 6 \\
\hline & Total & 7 (73 años) & 3 (69,6 años) & 10 \\
\hline \multirow{3}{*}{$\begin{array}{l}\mathrm{Si} \\
\mathrm{n}=39\end{array}$} & $\mathrm{Si}$ & 5 (75,2 años) & 3 (74 años) & 8 \\
\hline & No & 18 (77 años) & 13 (75 años) & 31 \\
\hline & Total & $\begin{array}{r}23(76,6 \\
\text { años) }\end{array}$ & 16 (75 años) & 39 \\
\hline \multicolumn{5}{|c|}{ Mejoría de la Ts } \\
\hline \multirow{3}{*}{$\begin{array}{l}\text { No } \\
\mathrm{n}=17\end{array}$} & $\mathrm{Si}$ & 2 (76 años) & 1 (67 años) & 3 \\
\hline & No & 7 (74 años) & 7 (74 años) & 14 \\
\hline & Total & 9 (74,4 años) & 8 (73,2 años) & 17 \\
\hline
\end{tabular}

\begin{tabular}{|c|c|c|c|c|}
\hline \multicolumn{5}{|c|}{$\begin{array}{c}\text { Tabla } 2 \\
\text { Resultados del análisis de regresión lineal }\end{array}$} \\
\hline & \multicolumn{4}{|c|}{ Variables a explicar } \\
\hline \multirow{2}{*}{$\begin{array}{l}\text { Variables } \\
\text { explicativas }\end{array}$} & \multicolumn{2}{|c|}{ MEVA } & \multicolumn{2}{|l|}{ MTS } \\
\hline & Coeficiente $\beta$ & $\mathrm{p}$ & Coeficiente $\beta$ & $\mathrm{p}$ \\
\hline EVAPREV & 0,441 & 0,000 & & \\
\hline TSPREV & & & $-0,528$ & 0,004 \\
\hline Constante & $-0,613$ & 0,301 & 0,414 & 0,000 \\
\hline $\mathrm{F}$ & 17,992 & 0,000 & 9,157 & 0,004 \\
\hline $\mathrm{N}$ & 49 & & 49 & \\
\hline $\mathrm{R}^{2}$ corregida & 0,261 & & 0,145 & \\
\hline \multicolumn{5}{|c|}{ Con fractura previa } \\
\hline EVAPREV & 0,455 & 0,000 & & \\
\hline TSPREV & & & $-0,372$ & 0,101 \\
\hline Constante & $-0,724$ & 0,386 & 0,388 & 0,000 \\
\hline $\mathrm{F}$ & 9,251 & 0,005 & 2,875 & 0,101 \\
\hline $\mathrm{N}$ & 30 & & 30 & \\
\hline $\mathrm{R}^{2}$ corregida & 0,221 & & 0,061 & \\
\hline \multicolumn{5}{|c|}{ Sin fractura previa } \\
\hline EVAPREV & 0,419 & 0,011 & & \\
\hline TSPREV & & & $-0,750$ & 0,022 \\
\hline Constante & $-0,430$ & 0,624 & 0,462 & 0,005 \\
\hline $\mathrm{F}$ & 8,067 & 0,011 & 6,385 & 0,004 \\
\hline $\mathrm{N}$ & 19 & & 19 & \\
\hline $\mathrm{R}^{2}$ corregida & 0,282 & & 0,230 & \\
\hline
\end{tabular}

\begin{tabular}{|l|c|c|c|c|c|c|}
\hline \multicolumn{7}{|c|}{ Tabla 3 } \\
\hline \\
\cline { 1 - 7 }
\end{tabular}


Los resultados de la regresión logística (tabla 3) obtenidos para una MEVA $>2$, MEVA $>3$ y MEVA $>4$ puntos, indican que estas mejorías de la EVA sólo se explican por la variable explicativa EVAPREV.

A medida que aumenta la EVAPREV, hay probabilidad de obtener una mejoría de la EVA en todos los casos (MEVA $>2, \operatorname{MEVA}>3$, MEVA>4), y la probabilidad de lograr mejorías más altas es mayor. Así, para el total de pacientes, un aumento de la EVAPREV en 1 punto, respecto a una EVAPREV de 0 puntos, tiene una probabilidad de mejorar la EVA, en más de 2 puntos, menor $(\mathrm{OR}=1,695$; $\mathrm{p}$ valor $=0,008)$, que de mejorar en más de 3 puntos $(\mathrm{OR}=2,021$; $\mathrm{p}$ valor $=0,006)$, y ambas menor que de mejorar en más de 4 puntos $(\mathrm{OR}=3,27 ; \mathrm{p}$ valor $=0,058)$. Aunque en este último caso $(\mathrm{MEVA}>4)$ no fue significativo al $95 \%$.

La regresión logística para las fracturas indica que la probabilidad de riesgo de fractura posterior, para el conjunto de pacientes, no se explica con la duración del tratamiento [OR=0,804 (IC 95\%: 0,64-1,01)].

\section{DISCUSIÓN}

En este primer trabajo en España sobre el efecto de la TPTD en los pacientes osteoporóticos, se muestra una reducción importante del dolor y una mejoría de la calidad de vida, así como una reducción también del número de fracturas, al igual que han demostrado los estudios realizados en otros países de Europa ${ }^{5,6,10,21,23,24}$, EE.UU ${ }^{18,20}$ y Japón ${ }^{13}$. El porcentaje de pacientes que experimentaron mejora de la calidad de vida (65\%) fue ligeramente inferior que los que obtuvieron mejora del dolor $(80 \%)$, debido a que se trata de pacientes con edad avanzada y la TS incluye además del dolor otras dimensiones.

No obstante, aunque se utilizó la misma escala de medida del dolor y de la calidad de vida que en los estudios anteriores ${ }^{5,6,10,11,23,24}$, la EVA y el EQ-5D, este trabajo está enfoca- do de forma novedosa, dado que analiza la mejora de cada paciente, y no solo en una muestra de pacientes, teniendo en cuenta su estado inicial y por ello, resulta difícil hacer una comparación exacta.

Así, en un trabajo publicado en diferentes medios ${ }^{5,6,10,23,24}$ en el que participaron 8 países europeos y utilizaron una muestra de 1.581 mujeres postmenopáusicas, se recogió simultáneamente los efectos de la TPTD durante 18 meses de tratamiento y 18 meses después del mismo, observándose una reducción significativa del dolor de espalda y de la incidencia de fracturas, y una mejoría en la calidad de vida, con la administración de la TPTD.

En nuestro trabajo, los resultados de la regresión lineal (tabla 2) indican que el estado inicial del paciente, medido por la EVAPREV y la TSPREV explican la mejoría del paciente. Además, tanto la regresión logística (tabla 3) como la regresión lineal, demuestran que la TPTD tiene un efecto significativamente mayor en pacientes con peor estado de salud inicial; esto es, permite a los pacientes con peores condiciones clínicas, lograr mayores incrementos de salud, lo que puede ser utilizado como criterio para las decisiones terapéuticas. El resto de las variables explicativas utilizadas (EVA posterior, TS posterior, duración del tratamiento, edad, existencia y tipo de fracturas) en el análisis no salieron significativas $(p>0,05)$. Esto significa que no se encontró ningún efecto de la duración del tratamiento (18 y 24 meses) en la mejora del dolor y la calidad de vida, al contrario que en trabajos previos. Así, en unos ${ }^{5,6,10,23,24}$ se midió el efecto de la TPTD en una muestra de pacientes en varios momentos a lo largo del periodo de administración (18 meses) y en los 18 meses posteriores, deduciéndose una mayor mejoría de la EVA a partir de los 18 meses, una vez finalizado el tratamiento, que prácticamente se mantuvo en los 18 meses posteriores. Esto ratifica una duración mínima de 18 meses de tratamiento, que podría ser ampliado a 24 meses $^{19}$, tal y como se 
ha procedido en nuestro trabajo. En otro estudio ${ }^{18}$ se midió igualmente, en una muestra de 1.637 mujeres postmenopáusicas procedentes de 17 países, el efecto de la TPTD en tres intervalos de duración de tratamiento: de 0 a 7 meses, de 7 a 14 y más de 14 meses. Encontraron una reducción de la fractura no vertebral, del dolor de espalda y de los efectos secundarios, en una duración más larga de administración de TPTD.

Tampoco se encontró en nuestro trabajo ninguna influencia significativa de la edad en la mejora del dolor y la calidad de vida, a pesar de que en la tabla 1 se puede observar que los grupos con mejoría de la EVA y de la TS tenían una edad media, entre 2 y 6 años, mayor que los grupos que no experimentaron dicha mejoría.

Igualmente, con la regresión, la existencia de fractura previa no explicó la mejoría del paciente. No obstante, según la tabla 2, en el grupo de pacientes con factura previa, se obtuvo un coeficiente de la variable EVAPREV más favorable, de 0,455 frente a 0,419 en el grupo sin fractura previa, en la explicación de la MEVA, y lo mismo ocurre en la variable TSPREV $(-0,372$ frente a $-0,750)$ en la explicación de la MTS. Los resultados de la regresión logística corroboran este aspecto en la MEVA $>2$ y MEVA $>3$, pero no en la MEVA $>4$.

En lo que respecta al riesgo de fracturas tras la administración de la TPTD, sí se pudo comprobar una reducción del número, como ocurre en otros trabajos previos ${ }^{5,6,10,20,21,23,24}$, superior al 50\%, (30 a 12 pacientes con fracturas) (tabla 1) y mayoritariamente vertebrales. Así, en un estudio ${ }^{20}$ se redujo el riesgo en todo tipo de fracturas (vertebrales y no vertebrales), en otro ${ }^{21}$ se demostró la reducción del riesgo de fracturas vertebrales, en 217 pacientes frente a 106 con tratamiento estándar (bifosfonatos), a lo largo de 5 años. Las conclusiones fueron una reducción sustancial del riesgo de fractura y una preferencia de este tratamiento en aquellos pacientes con osteoporosis severa vertebral, debido a la superioridad en la prevención de nuevas fracturas de este tipo. Otro estudio ${ }^{22}$, realizado igualmente con pacientes mayores (edad media 73,4+5,8 años), mostró que la TPTD no sólo redujo la incidencia de nuevas fracturas vertebrales en un $96,5 \%$ y consiguió aumentos del $12 \%$ de la densitometría lumbar y $11 \%$ en cuello femoral, sino que además redujo en un $80 \%$ el consumo de anti-inflamatorios no esteroideos.

Pero en nuestro trabajo no se apreció diferencias en la reducción del riesgo de fracturas, entre los pacientes que habían sufrido, o no, una fractura antes del inicio del tratamiento, y tampoco entre las distintas edades, como se aprecia en la tabla 1. Igualmente, según la tabla 3 , no se pudo explicar esta reducción con el estado inicial del paciente, ni con la duración del tratamiento (pvalor $>0,05$ para el conjunto de pacientes), lo cual quiere decir que no existen diferencias entre una duración de 18 y 24 meses, al igual que ocurrió en otro estudio previo ${ }^{21}$, lo que hace dudar de la necesidad de ampliar el tratamiento a 24 meses.

Esto se contradice con otro estudio 4 que demostró, con un total de 761 miembros de Medicare que, tras la administración de TPTD a lo largo de 12 meses, aquellos que habían tenido una fractura en los 6 meses antes del inicio del Tratamiento (50\%), a los 12 meses tenían una probabilidad 3 veces mayor de sufrir una fractura.

Finalmente, independientemente de la mejoría, o no, de la EVA y de la TS, los pacientes que no sufrieron fractura antes del tratamiento tenían una edad media menor que los que sí la sufrieron (tabla 1), lo cual ratifica que la edad es un factor de riesgo de fractura en pacientes osteoporóticos como indican otros trabajos ${ }^{7}$. Pero independientemente de que presentaran fractura previa, o no, los pacientes que mejoraron la EVA tras el tratamiento (16 sin fractura previa y 23 con fractura previa), tuvieron mayor edad media (75 y 76,6 años), que los que no experimentaron esa mejora ( 3 sin fractura previa 
y edad media de 69,6 años, frente a 7 con fractura previa y edad media de 73 años).

Este presente estudio tiene algunas limitaciones. En primer lugar, no hay un grupo de comparación de pacientes, sólo se tienen los resultados para los pacientes a los que se les administró la TPTD, y tampoco se pudo comparar su efecto con otros fármacos alternativos. En segundo lugar, la duración del tratamiento sólo fue de 18 y 24 meses, y no fue posible analizar el efecto a duraciones menores.

El punto fuerte de nuestro estudio es que se analizó la mejoría de cada paciente y se midió esa mejoría en relación al estado de salud inicial.

En un futuro sería interesante aumentar el tamaño de la muestra, ampliar el periodo de estudio e introducir nuevas variables similares a las consideradas en los estudios previos (clínicas, hábitos de vida, etc.) con el fin de poder realizar comparaciones más precisas, incluir la influencia de aspectos clínicos, los tipos de fractura y mejorar la fiabilidad de los resultados para identificar las posibles características clínicas predictoras de respuesta al tratamiento.

\section{BIBLIOGRAFÍA}

1. Wade SW, Satram-Hoang S, Nadkar A, Macarios D, Tosteson A. Impact of Medication Adherence on Health Care Utilization and Productivity: Self-Reported Data From a Cohort of Postmenopausal Women on Osteoporosis Therapy. Clin Ther. 2011; 33(12): 20062015.

2. Arana-Arri E, Gutiérrez-Ibarluzea I, Ecenarro Mugaguren A, Asua Batarrilta J. Prevalencia de ciertos hábitos determinantes de osteoporosis en mujeres postmenopáusicas del País Vasco en 2003. Rev Esp Salud Pública. 2007; 81: 647-656.

3. Quattrocchi E, Kourlas H. Teriparatide: A Review. Clin Ther. 2004; 26(6): 841-854.

4. Hazel-Fernandez L, Louder AM, Foster SA, Uribe CL, Burge RT. Association of teriparatide adherence and persistence with clinical and economic outcomes in Medicare Part D recipients: a retrospective cohort study. BMC Musculoskeletal Disord. 2013; 14(4): $2-17$.
5. Fahrleitner-Parmmer A, Langdahl BL, Marin F, Jakob F, Karras D, Barrett A, et al. Fracture rate and back pain during and after discontinuation of teriparatide: 36 months data from the European Forsteo Observational Study (EFOS). Osteoporos Int. 2011; 22:27092719.

6. Walsh JB, Lems WF, Karras D, Langdahl BL, Ljunggren $\mathrm{O}$, Fahrleitner-Pammer A, et al. Effectiveness of Teriparatide in Women Over 75 Years of Age with Severe Osteoporosis: 36-Month Results from the European Forsteo Observational Study (EFOS). Calcif Tissue Int. 2012; 90:373-383.

7. Mesa Ramos M, editor. Guía de práctica clínica osteoporosis. Grupo de Estudio e Investigación de la Osteoporosis de la Sociedad Española de Cirugía Ortopédica y traumatología. 2010. Madrid: Multimédica Proyectos SL;2010.

8. Álvarez J. Prevención de la osteoporosis. El costo de la desinformación del paciente. Econ Salud. 2002; 1(4):24-26.

9. Gómez Navarro R. Prevalencia de los factores de riesgo de fractura por fragilidad en varones de 40 a 90 años de una zona básica de salud rural. Rev Esp Salud Pública. 2011;85: 491-498.

10. Rajzbaum G, Grados F, Evans D, Liu-Leage S, Petto H, Augendre-Ferrante B. Treatment persistence and changes in fracture risk, back pain, and quality of life amongst patients treated with teriparatide in routine clinical care in France: Results from the European Forsteo Observational Study. Joint Bone Spine. 2014; 81:69-75.

11. Parthan A, Kruse M, Agodoa I, Silverman S, Orwoll E. Denosumab: A cost-effective alternative for older men with osteoporosis from a Swedish payer perspective. Bone. 2014; 59:105-113.

12. Saito M, Marumo K, Kida Y, Ushiku C, Kato S, Takao-Kawabata $\mathrm{R}$, et al. Changes in the contents of enzymatic immature, mature, and non-enzymatic senescent cross-links of collagen after once-weekly treatment with human parathyroid hormone (1-34) for 18 months contribute to improvement of bone strength in ovariectomized monkeys. Osteoporos Int. 2011; $22: 2373-2383$.

13. Tsujimoto M, Uenaka K, Iwata A, Higashiuchi Y, Sowa H. Effects of teriparatide in Japanese and nonJapanese populations: bridging findings on pharmacokinetics and efficacy. J Bone Miner Metab. 2012; 30:326-337.

14. Ohbayashi Y, Miyake M, Sawai F, Minami Y, Iwasaki A, and Matsui Y. Adjunct teriparatide therapy with monitoring of bone turnover markers and bone scintigraphy for bisphosphonate-related osteonecrosis of the jaw. Oral Med. 2013; 115(4): e31-e37. 
15. Saag Kg, Zanchetta JR, Devogelaer JP, Adler RA, Eastell R, See K, et al. Effects of teriparatide versus alendronate for treating glucocorticoid-induced osteoporosis: thirty-six-month results of a randomized, double-blind, controlled trial. Arthritis Rheum. 2009; 60(11): 33463355 .

16. Iolascon G, Gimigliano F, Resmini G. Teriparatide and orthopedic surgery. Aging Clin Exp Res. 2007; 19 (Suppl): 22-25.

17. Pietrogrande L, Raimondo E. Teriparatide in the treatment of non-unions: Scientific and clinical evidence. Injury, Int. J. Care Injured. 2013; 44: S54-S57.

18. Lindsay RP, Miller G, Pohl E, Glass VP, Chen J, Krege H. Relationship between duration of teriparatide therapy and clinical outcomes in postmenopausal women with osteoporosis. Osteoporos Int. 2009; 20:943-948.

19. Obermayer-Pietsch BM, Marin F, McCloskey EV, Hadji P, Farrerons J, Boonen S, et al. Effects of two years of daily teriparatide treatment on BMD in postmenopausal women with severe osteoporosis with and without prior antiresorotive treatment. J Bone Miner Res. 2008; 23(10): 1591-1600.

20. Yu S, Burge RT, Foster S, Gelwicks SA, Meadows ES. The impact of teriparatide adherence and persistence on fracture outcomes. Osteoporos Int. 2012; 23:11031113 .

21. Oswald AJ, Berg J, Milne G, Ralston SH. Teriparatide Treatment of Severe Osteoporosis Reduces the Risk of Vertebral Fractures Compared with Standard Care in Routine Clinical Practice. Calcif Tissue Int. 2014; 94:176-182.

22. Maugeri D, Russo E, Luca S, Leotta C, Mamazza G, Sorace R, et al. Changes of the quality-of-life under the treatment of severe senile osteoporosis with teriparatide. Arch Gerontol Geriatr. 2009; 49(1): 35-38.

23. Jakob F, Oertel H, Langdahl B, Ljunggren O, Barret A, Karras D, et al. Effects of teriparatide in postmenopausal women with osteoporosis pre-treated with bisphophonates: 36-month results from the European Forsteo Observational Study. Eur J Endocrinol. 2012; 166(1):8797.

24. Ljunggren Ö, Barrett A, Stoykov I, Langdahl BL, F Lems WF, Walsh JB, et al. Effective osteoporosis treatment with teriparatide is associated with enhanced quality of life in postmenopausal women with osteoporosis: the European Forsteo Observational Study. BMC Musculoskeletal Disord. 2013;14: 251.

25. Instituto Nacional de Estadística. Cifras de población. Madrid: INE;2014. Disponible en: www.ine.es.

26. Badia X, Carné X. La evaluación de la calidad de vida en el contexto de ensayo clínico. Med Clin. (Barc). 1998; 110:550-556.
27. Herdman M, Badia X, Berra S. El EuroQol-5D: una alternativa sencilla para la medición de la calidad de vida relacionada con la salud en atención primaria. Aten Prim. 2001; 28(6): 425-430.

28. Polyzos SA, Anastasilakis AD, Bratengeier C, Woloszczuk W, Papatheodorou A, Terpos E. Serum sclerostin levels positively correlate with lumbar spinal bone mineral density in postmenopausal women - the sixmonth effect of risedronate and teriparatide. Osteoporos Int. 2012; 23:1171-1176.

29. Anastasilakis A, Polyzos S, Makras P, Gkiomisi A, Bisbinas I, Katsarou A, et al. Circulating irisin is associated with osteoporotic fractures in postmenopausal women with low bone mass but is not affected by either teriparatide or denosumab treatment for 3 months, Osteoporos Int. 2014; 25(5): 1633-1642. 